\title{
Automated Microscopy to Evaluate Surgical Specimens Via Touch Prep in Breast Cancer
}

\author{
Maria Jose Cortes-Mateos, PhD ${ }^{1}$, David Martin, $\mathbf{M S}^{2}$, Sergio Sandoval, $\mathbf{M S}^{3}$, Manuel E. Ruidiaz, $\mathbf{M S}^{3}$, \\ Davorka Messmer, $\mathrm{PhD}^{1}$, Jessica Wang-Rodriguez, $\mathrm{MD}^{4}$, William Trogler, $\mathrm{PhD}^{5}$, Andrew C. Kummel, $\mathrm{PhD}^{5}$, \\ and Sarah L. Blair, MD ${ }^{6}$
}

\begin{abstract}
${ }^{1}$ Moores Cancer Center, University of California San Diego, San Diego, CA; ${ }^{2}$ Department of Electrical and Computer Engineering, University of California San Diego, San Diego, CA; ${ }^{3}$ Department of Bioengineering, University of California San Diego, San Diego, CA; ${ }^{4}$ Department of Pathology, University of California San Diego, San Diego, CA; ${ }^{5}$ Department of Chemistry and Biochemistry, Nanoengineering University of California San Diego, San Diego, CA; ${ }^{6}$ Department of Surgery, Moores Cancer Center, University of California San Diego, San Diego 92093-0987, CA
\end{abstract}

\begin{abstract}
Background. Breast conservation therapy is the standard treatment for breast cancer; however, $20-50 \%$ of operations have a positive margin leading to secondary procedures. The standard of care to evaluate surgical margins is based on permanent section. Imprint cytology (touch prep) has been used to evaluate surgical samples, but conventional techniques require an experienced cytopathologist for correct interpretation. An automated image screening process has been developed to discern cancer cells from normal epithelial cells. This technique is based on cellularity of the imprint specimen and does not require expertise in cytopathology.

Methods. A rapid immunofluorescent staining technique coupled with automated microscopy was used to classify specimens as cancer vs. noncancer based on the density of epithelial cells captured on touch prep of tumor crosssections. The results of the automated analysis vs. a manual screen of ten $20 \times$ fields were compared to the pathology interpretation on permanent section.

Results. A total of 34 consecutive cases were analyzed: 10 normal cases, and 24 cancer cases. The cross-section specimens for invasive cancer were correctly classified in at least $65 \%$ of the cases by using manual microscopy and at least $83 \%$ by using automated microscopy. The manual and automated microscopy correlated well for
\end{abstract}

(C) The Author(s) 2009. This article is published with open access at Springerlink.com

First Received: 22 August 2008;

Published Online: 10 January 2009

S. L. Blair, MD

e-mail: slblair@ucsd.edu measurements of epithelial cell density $\left(R^{2}=0.64\right)$; however, the automated microscopy was more accurate.

Conclusions. This preliminary study using an automated system for intraoperative interpretation does not require a cytopathologist and shows that rapid, low-resolution imaging can correctly identify cancer cells for invasive carcinoma in surgical specimens. Therefore, automated determination of cellularity in touch prep is a promising technique for future margin interpretation of breast conservation therapy.

Multiple, randomized, prospective trials with greater than a 10-year follow-up have proven that breast conservation therapy (BCT) has equal survival efficacy compared with mastectomy in treating early-stage breast cancer; therefore, $\mathrm{BCT}$ has become the standard of care to treat this malignancy. ${ }^{1-4}$ Another important aspect of BCT is improved cosmetic outcome, because the best results are obtained at the time of the initial operation with a single excision and better aesthetic outcomes correlate with improved quality of life. ${ }^{5}$ However, obtaining a negative margin in localized excision with primary BCT is still a challenge. Many studies show that local recurrence is significantly higher in patients with a positive margin vs. a negative margin excision ${ }^{3,6,7}$. Despite improved preoperative imaging techniques, such as breast MRI and ultrasound, many studies report positive margin rates of $20-50 \%$ for partial mastectomy, even for patients with early-stage breast cancer. Therefore, achieving negative surgical margins is an essential goal in breast cancer treatment, because the consequences of a failed margin are significant for patients, surgeons, and the healthcare system. ${ }^{8}$ 
The "gold standard" for achieving negative margins for most tumors is performing multiple intraoperative frozen sections on the margins of the excised tumor. However, this technique has many limitations. Freezing the adipose tissue of the breast and preparing adequate and representative sections is a technically complex process. $^{9}$ Frozen-section evaluations on multiple samples from a three-dimensional cavity, while the patient and the surgeon are waiting for the results in the operating room, are extremely labor intensive and time consuming. In addition, there is concern that in small tumors the frozen sections may utilize the majority of the available tumor tissue and there will be insufficient specimens available for adequate histological evaluation and associated studies on permanent sections. The technique of using touch preps to detect tumor cells at breast cancer surgical margins has been studied during the last 20 years. ${ }^{10-12}$ The largest study by Klimberg et al. ${ }^{11}$ examined 428 patients with breast lesions and had a sensitivity of $96 \%$ and specificity of $100 \%$. This group performed scrape and touch preps of a cross-section of the tumor as well as the marginal tissue around the tumor. The slides were fixed, stained with hematoxylin and eosin, and examined by an experienced cytopathologist. However, other institutions have not embraced this technique because artifacts associated with air drying and surface cautery can affect touch prep interpretation and the availability of an experienced cytopathologist limits its widespread use. ${ }^{9}$ The objective of this project is to develop an automated real-time intraoperative technique to detect cancer cells present at surgical margins to prevent positive margins and the need for a second operation to clear the microscopic margins. The long-term goal is to reduce the necessity of multiple operations to surgically treat breast cancer.

\section{METHODS}

\section{Surgical Samples}

Institutional Review Board approval from the University of California, San Diego, was obtained to study breast cancer and noncancer tissue from patients. All patients underwent their planned procedure for breast surgical treatment, and the specimens were removed and sent to the pathology laboratory for analysis. Thirty-four consecutive cases, both cancerous $(n=24)$ and normal tissue $(\mathrm{n}=10)$, were studied. The normal tissue was obtained from patients who underwent breast reduction or prophylactic mastectomy. All surgeries were performed at the University of California, San Diego. Standard techniques for resection of breast tissue were used, including electric cautery (Valley Labs, Boulder, CO). Fresh specimens were gently imprinted onto poly-L-lysine (PLL) coated glass slides (Newcomer Supply, Middleton, WI).

\section{Cross-Sections}

To study the efficacy of the proposed technique in correctly identifying cancer cells in fresh surgical specimens by immunofluorescence (IF), the excised tissue was cut through the center to reveal the tumor (designated as "cross-section"), and a touch prep of the cross-section was performed. The surgical specimens were grossed in by a surgical pathologist to locate the area with the highest probability of identifying tumor cells. These cross-sectional cuts do not have a cauterized surface making them ideal specimen to establish the reliability and accuracy of the automated analysis technique on the most consistent available samples. The tissue was bisected and immediately imprinted/touched onto PLL slides to minimize airdrying artifacts. For invasive carcinomas, the location of the tumor in the tissue was usually located by palpation; therefore, the cross-section nearly always bisected the tumor. For smaller tumors, such as ductal carcinoma in situ (DCIS), the location of the tumor was approximated by mammographic needle localization. However, many of the small tumors had been subjected to a previous core biopsy, which may have removed most of the cancer cells. The mammographers at University of California, San Diego, routinely use 9- to 11-gauge vacuum-assisted cores to establish a diagnosis.

\section{Immunofluorescence Staining}

Two quantifiers were used to identify the cancer cells: (1) cytokeratin was used as a specific marker for epithelial cells to distinguish them from other cells that can be found attached to the PLL-slide after performing touch prep (leukocytes, adipocytes, fibroblasts, etc.); (2) cell density was quantified by using Hoechst 33342 (Invitrogen, Carlsbad, CA) as a nuclear dye. The Hoechst staining served several purposes: it enabled the exclusion of nonnucleated cells (e.g., erythrocytes), and facilitated counting the cells within clusters (Fig. 1). Mouse IgG isotype control (Southern Biotechnology, Birmingham, AL) and anticytokeratin antibody (DakoCytomation, Carpinteria, CA) were labeled using the Alexa Fluor 488 Zenon Mouse IgG Labeling Kit (Invitrogen) following the manufacturer's instruction and kept in the dark at $4{ }^{\circ} \mathrm{C}$.

The slides were fixed with $4 \%$ paraformaldehyde (PFA) in phosphate-buffered saline (PBS) for $10 \mathrm{~min}$ at room temperature and rinsed by immersion in PBS. To reduce background from nonspecific binding of the antibodies to the cell Fc receptors, the samples were incubated at room 
FIG. 1 Immunofluorescence staining of touch-prep slides. Individual cells, small cell clusters, and big cell clusters can be observed on the slide. Based on the number of epithelial cells and/or clusters, the slides are classified as positive or negative
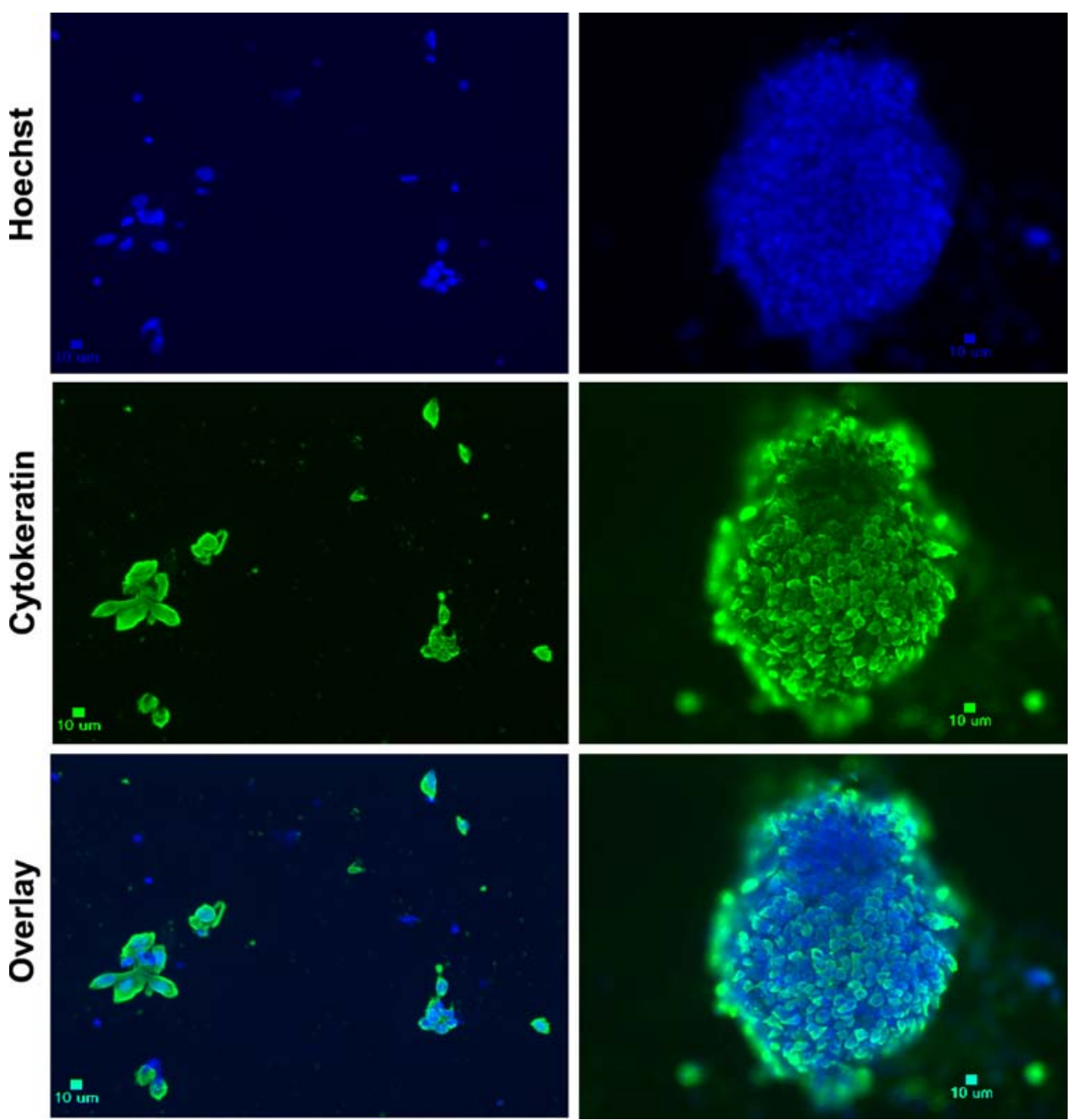

temperature for $5 \mathrm{~min}$ with human $\operatorname{IgG}(10 \mu \mathrm{g} / \mathrm{ml}$; Invitrogen) in PBS followed by washing by immersion in PBS. After blocking, the samples were permeabilized and stained in one step using the Inside Stain Kit (Miltenyi Biotec, Aubum, CA). AlexaFluor 488 labeled antibodies (mouse $\mathrm{IgG}$ isotype control and anti-cytokeratin) were diluted with Inside Perm reagent at $6 \mu \mathrm{g} / \mathrm{ml}$ and Hoechst at $10 \mu \mathrm{g} / \mathrm{ml}$. The samples were incubated in the permeabilization-staining solution for $15 \mathrm{~min}$ at room temperature in the dark. Afterwards, the samples were washed by immersion in PBS. Finally, the slides were mounted with ProLong Gold (Invitrogen), a microscope cover glass was placed on the slide, and the slide was analyzed by microscopy. The complete staining protocol, including fixing, permeabilization, and blocking took only 20-25 $\mathrm{min}$.

\section{Manual Analysis of the Slides}

After staining, the slides were analyzed manually using a fluorescent microscope (Nikon Eclipse E600, Nikon Instruments Inc., Melville, NY) with a triple bandpass filter
(DAPI/FITC/Texas Red w/Single-Band Exciters, Chroma Technology Corp, Rockingham, VT). Ten fields close to the centerline of the slide were chosen and analyzed using a $20 \times$ objective. The total number of cells was obtained by counting all nuclei (Hoechst positive), and the number of epithelial cells was obtained by counting cytokeratinpositive cells. Large three-dimensional clusters of epithelial cells were considered cancerous. The cell count for each large cluster was estimated by counting the number of nuclei in a fraction of each cluster and then correcting for the total area of the cluster. From the ten fields of view at $20 \times$ along the centerline of the long axis of the slide, both the number of epithelial cells $/ \mathrm{mm}^{2}$ (epithelial cell density) and the percentage of epithelial cells were calculated. It is noted that in this manual microscopy method, the choice of image fields was focused on the area of highest concentration of epithelial cells. The microscopist examined a progression of ten locations along the centerline. At each location, the field of view was adjusted in the local region $( \pm 300 \mu \mathrm{m})$ to image as many cells as possible. Therefore, it is expected that the manual microscopy method will 
measure a higher average epithelial cell density than the automated microscopy method, but there should be a strong correlation between the two measurements.

\section{Automated Microscopy and Analysis of the Slides}

An automated scanning stage microscope was used for the data acquisition (AxioImager Z1, Carl Zeiss Inc., Thornwood, NY). In the automated microscopy analysis, the entire surface of each touch prep slide was imaged at a magnification of $5 \times$ with a 1.4 megapixel camera (CoolSNAP $\mathrm{HQ}^{2}$, Photometrics, Pleasanton, CA); the absolute resolution was $1.267 \mu \mathrm{m} /$ pixel. A series of focus points for the slide was first determined manually along with appropriate exposure times for each fluorescent channel to maximize sensitivity to staining intensity. Afterwards, the automated microscope divided the slide into a series of tiles and recorded two fluorescent images (one for Hoechst fluorescence and one for cytokeratin fluorescence) at each of these tiles. Finally, the tiled images were loaded into a custom fluorescent image analysis program to specifically recognize both isolated cells and cells within tightly packed clusters. The cellular recognition software used an image tile of Hoechst fluorescence and an image tile of cytokeratin fluorescence at each location on the slide.

Each Hoechst image was used to determine how many nonepithelial cells were present in a given location and to filter out cytokeratin-positive artifacts. First, an estimation of background Hoechst fluorescence was determined for the image tile by sampling intensity values across $10 \%$ of the image tile. The mean and standard deviation of fluorescence intensity were calculated from this sampling set, and the intensity value one standard deviation below the mean was used as an estimate of background fluorescence for the image tile, and subtracted from all other pixels in that tile. Due to inherent variations of fluorescent staining across a slide, determining a distinct background value for each image tile was crucial. After background subtraction, median filtering was applied to the image tile to reduce grain noise while preserving edges. Next, contrast enhancement was performed to maximize the intensity difference between background staining and any positively stained cell nuclei in the image. Finally Otsu thresholding, a technique that enhances object outlines by separating foreground areas of an image from background areas, was performed on the image tile to obtain binary outlines for any nuclei present. ${ }^{13}$ After all image processing steps are complete, valid outlines of nuclei must be filtered out from any erroneous outlines of image artifacts. A Hoechst outline is determined to be a valid outline of a nucleus if its average Hoechst fluorescence is twice the background, the nuclear diameter is $>4.5 \mu \mathrm{m}$, and the circularity of the outline is $>0.6$ for which 1 represents a perfect circle.
Automated interpretation of cytokeratin fluorescence was more complicated. Epithelial cells tended to be captured by touch prep in large clusters of cells, which were poorly penetrated by the anti-cytokeratin antibody. Poor antibody penetration and cell layering effects in clusters made it difficult to develop a universal algorithm using standard methods that could identify both single epithelial cells and each individual epithelial cell located inside of a cell cluster. Instead an algorithm was developed that finds a single outline for each cluster of epithelial cells as well an outline for each isolated epithelial cell. (a) Background subtraction on a tile by tile basis, as described in the Hoechst image procedure, was performed on each cytokeratin tile image to normalize intensity values to any background staining and a median filter was applied to reduce grain noise. (b) A hole-filling algorithm was used to fill in any weakly stained areas between cells located in a cluster that would otherwise cause fragmented outlines. The hole-filling algorithm has no effect on single cells because it only smoothes fluorescent intensity across a cell's cytoplasm. (c) A Sobel edge enhancement-a technique that enhances edges in an image by differentiating the intensity gradient in a radius around each pixel-was performed to convert the image into a binary representation of the edges of all cells and clusters present. ${ }^{14}$ (d) The edge representation was slightly dilated to compensate for any information loss in the previous steps. (e) A hole-filling was performed once more before Otsu thresholding to determine final single epithelial cell and epithelial cell cluster outlines for an image. (f) The area occupied by each epithelial cell cluster was calculated. By dividing each cluster area by the average size of an epithelial cell $\left(200 \mu \mathrm{m}^{2}\right)$, an approximation for the total number of epithelial cells in each cluster was obtained (Fig. 2). Note that this method assumes all clusters occupy a two-dimensional plane, thereby undercounting the number of epithelial cells in multilayer clusters. Epithelial cell outlines were checked for validity before being used in the statistical analysis. Raw cytokerative outlines obtained from the image processing steps are filtered to have a nuclear area of at least $15 \mu \mathrm{m}^{2}$, an average cytokeratin fluorescence twice that of the background cytokeratin fluorescence of the image tile, and an average Hoechst fluorescence intensity greater than the background Hoechst intensity of the image tile to ensure accepted cytokeratin outlines contain cell nuclei and thus represent epithelial cells. The automated microscopy currently requires 45 min to scan an entire slide with a $5 \times$ objective and a 1.4 megapixel camera; with a 14 megapixel camera, this will be reduced to less than $5 \mathrm{~min}$. The current microscope requires 15 min of setup time, because it does not have a practical auto-focusing system. By using a larger camera, a lower magnification objective can be used to maintain a fixed imaging resolution, which will reduce the 

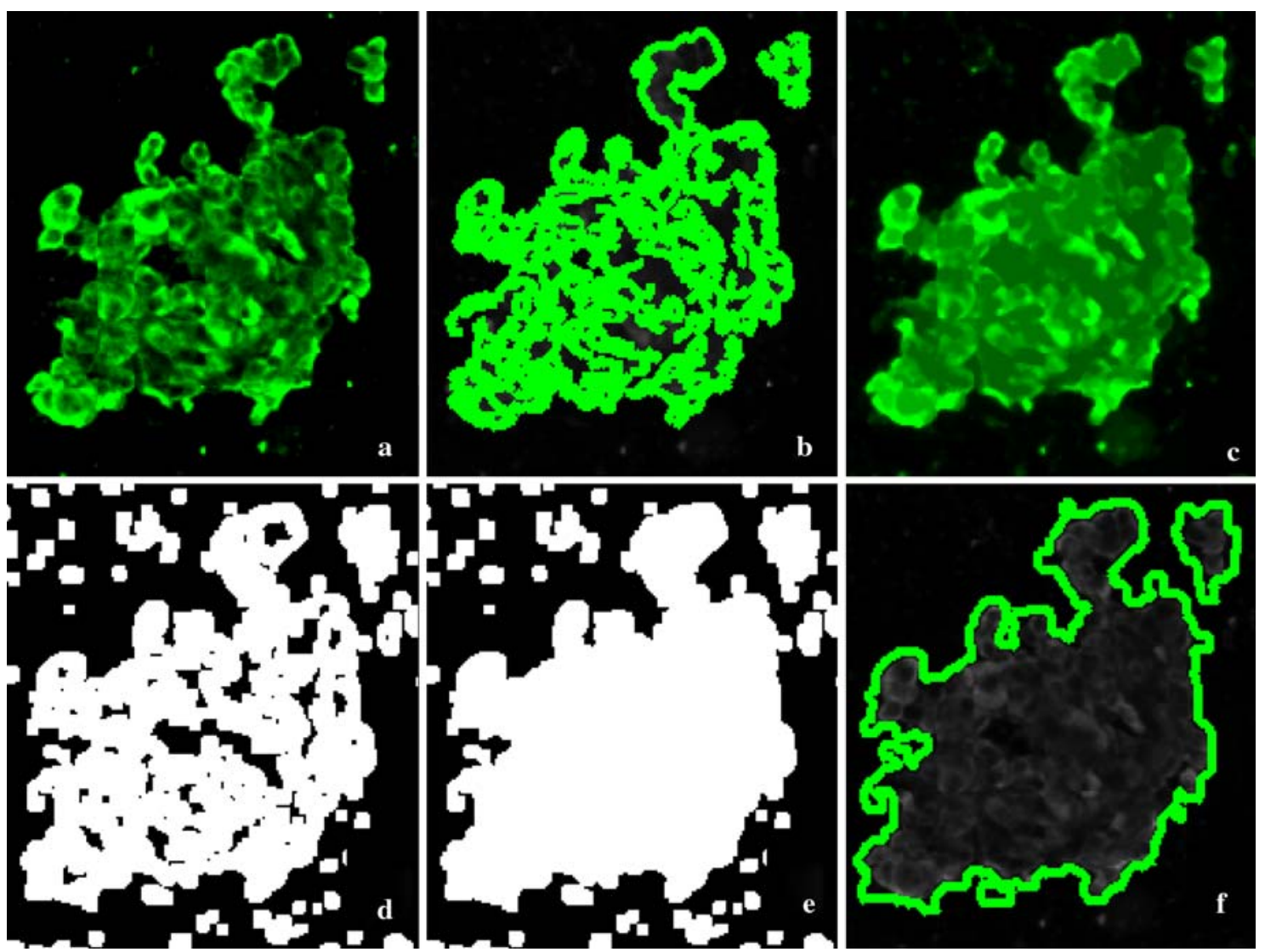

FIG. 2 Automatic microscopy algorithm. a Background subtracted cytokeratin fluorescence image tile. b Fragmented outlines resulting from standard outlining techniques. c Median filtering and hole

time required for auto focusing to less than 1 min with efficient software.

\section{Statistics}

Automated and manual microscopy were correlated with Pearson's correlation. Cutoff values between benign and malignant cells were calculated based on Fisher linear discriminant analysis. ${ }^{15}$

\section{RESULTS}

\section{Patient Profiles}

Thirty-four consecutive surgical samples were analyzed (from August 2007 to April 2008). Ten of these samples were noncancerous tissues ( 6 from breast reduction and 4 from prophylactic mastectomies), and 24 samples were breast cancer with differing diagnostic classifications (6 DCIS; 14 invasive ductal carcinoma (IDC); 1 lobular carcinoma in situ (LCIS), and 3 invasive lobular carcinoma (ILC)). The 14 cases of IDC were subclassified as follows: filling. d Sobel Edge enhancement and dilation. e Hole filling. f Final epithelial outlines overlain on original image tile

7 (50\%) were only IDC, 4 (29\%) were IDC/DCIS, 2 (14\%) were IDC with lobular features, and 1 (7\%) was IDC/LCIS.

Thirteen patients underwent mastectomies and 11 underwent lumpectomy or BCT (Table 1). One to five serial touch prep slides of cross-sections were analyzed for each sample. The touch prep results were compared to final evaluation of the entire specimen by the pathologists at the University of California, San Diego.

\section{Analysis of Multiple Cross-Sectional Cuts}

The density of epithelial cells in the cross-section slides of normal and cancer samples were compared by using the manual counting method vs. automated microscopy. An example of this technique is shown in Fig. 3a; manual microscopy for case $\mathrm{C} 14$ contained a $5-\times 2-\mathrm{cm}$ tumor in a $8-\times 6-\times 5-\mathrm{cm}$ tissue sample. Slices 1,2 , and 3 had an epithelial cells density more than tenfold greater than slices 4 and 5 , consistent with the touch prep method accurately locating the tumor. The edges of the tumor had fewer cells captured than the center of the tumor. To determine the probability of sampling the tumor and the expected variation of epithelial cell density along the total tissue sample, 
TABLE 1 Patients' demographic information, including age, diagnosis, and pathological data obtained from pathology laboratory

\begin{tabular}{|c|c|c|c|c|c|}
\hline Sample & Age (yr) & Surgery & Diagnosis & Grade & Stage \\
\hline $\mathrm{C} 1$ & 46 & $\mathrm{~L}$ & DCIS & 2 & 0 \\
\hline $\mathrm{C} 2$ & 58 & $\mathrm{~L}$ & DCIS & 2 & 0 \\
\hline $\mathrm{C} 3$ & 56 & M & DCIS & 3 & 0 \\
\hline $\mathrm{C} 4$ & 57 & M & DCIS & 2 & 0 \\
\hline $\mathrm{C} 5$ & 64 & M & DCIS & 3 & 0 \\
\hline C6 & 59 & $\mathrm{~L}$ & DCIS/LCIS & 3 & 0 \\
\hline $\mathrm{C} 7$ & 49 & $\mathrm{~L}$ & IDC & 2 & I \\
\hline $\mathrm{C} 8$ & 49 & $\mathrm{~L}$ & IDC & 3 & III \\
\hline C9 & 52 & $\mathrm{~L}$ & IDC & 3 & I \\
\hline $\mathrm{C} 10$ & 70 & $\mathrm{~L}$ & IDC & 3 & I \\
\hline $\mathrm{C} 11$ & 42 & M & IDC & 2 & I \\
\hline $\mathrm{C} 12$ & 49 & M & IDC & 3 & III \\
\hline $\mathrm{C} 13$ & 86 & M & IDC & 2 & II \\
\hline $\mathrm{C} 14$ & 58 & $\mathrm{~L}$ & IDC/DCIS & 2 & II \\
\hline $\mathrm{C} 15$ & 59 & $\mathrm{~L}$ & IDC/DCIS & 2 & I \\
\hline $\mathrm{C} 16$ & 41 & M & IDC/DCIS & 3 & I \\
\hline $\mathrm{C} 17$ & 58 & M & IDC/DCIS & 2 & I \\
\hline $\mathrm{C} 18$ & 48 & $\mathrm{~L}$ & IDC/ILC & 2 & III \\
\hline C19 & 68 & $\mathrm{~L}$ & IDC/ILC & 1 & II \\
\hline $\mathrm{C} 20$ & 40 & M & IDC/LCIS & 1 & I \\
\hline $\mathrm{C} 21$ & 49 & M & LCIS & 2 & 0 \\
\hline $\mathrm{C} 22$ & 40 & M & ILC & 2 & II \\
\hline $\mathrm{C} 23$ & 56 & M & ILC & 2 & III \\
\hline $\mathrm{C} 24$ & 49 & M & ILC & 2 & I \\
\hline N1 & 18 & $\mathrm{BR}$ & $\mathrm{N}$ & & \\
\hline $\mathrm{N} 2$ & 23 & $\mathrm{BR}$ & $\mathrm{N}$ & & \\
\hline N3 & 26 & $\mathrm{BR}$ & $\mathrm{N}$ & & \\
\hline N4 & 30 & $\mathrm{BR}$ & $\mathrm{N}$ & & \\
\hline N5 & 47 & $\mathrm{BR}$ & $\mathrm{N}$ & & \\
\hline N6 & 71 & $\mathrm{BR}$ & $\mathrm{N}$ & & \\
\hline N7 & 56 & PM & $\mathrm{N}$ & & \\
\hline N8 & 41 & PM & $\mathrm{N}$ & & \\
\hline N9 & 42 & PM & $\mathrm{N}$ & & \\
\hline N10 & 43 & $\mathrm{PM}$ & $\mathrm{N}$ & & \\
\hline
\end{tabular}

$L$ lumpectomy, $M$ mastectomy, $B R$ breast reduction, $P M$ prophylactic mastectomy, DCIS ductal carcinoma in situ, IDC invasive ductal carcinoma, LCIS lobular carcinoma in situ, ILC invasive lobular carcinoma, $N$ normal

serial cross-section sampling (with 2 to 5 serial cuts) was performed in 20 samples (13 cancer and 7 normal samples). To estimate the variation of epithelial cell density between all cuts for a given tissue sample, the minimum number of cells per $\mathrm{mm}^{2}$ on a single cut was divided by maximum number of cells per $\mathrm{mm}^{2}$ on a single cut. As shown in Fig. 3b, most normal cases have a ratio close to 1 . In three normal cases, the ratio was lower, which was attributed to high adipose breast tissue in patients who underwent breast reduction operations. For IDC and DCIS, the ratio of maximum to minimum epithelial cells can be very low, consistent with a high probability of missing the tumor in a single random cut.

For invasive tumors, the probability of bisecting the tumor in a cross-section tissue cut was high, because the tumors studied were usually palpable and large (average tumor size in $\mathrm{cm}$ : $\mathrm{IDC}=2 \pm 1.3$; $\quad$ ILC $=3.1 \pm 2.7$ ). Conversely, noninvasive carcinomas are more difficult to sample in cross-sectional tissue cuts because these tumors are smaller (DCIS $=1.1 \pm 0.7 \mathrm{~cm}$ ) and rarely palpable. To determine the accuracy of the tumor detection technique, the probability of missing the tumor in a crosssectional cut in the absence of palpation was estimated from the ratio of Feret's diameters of the tumors and tissue. Feret's diameter is the longest distance between any two points of an outline. For a single slice, the probability of missing the tumor $(p)$ is a linear function of the relative diameters of the tissue $\left(\mathrm{d}_{\text {tissue }}\right)$ and the tumor $\left(\mathrm{d}_{\text {tumor }}\right)$ : $\mathrm{p}=1-\mathrm{d}_{\text {tumor }} / \mathrm{d}_{\text {tissue }}$. When multiple slices are taken, the probability of missing the tumor is lowered as a power function of the number of slices (n). Probability of missing the tumor for $\mathrm{n}$ slices $=\mathrm{p}^{\mathrm{n}}=\left(1-\mathrm{d}_{\text {tumor }} / \mathrm{d}_{\text {tissue }}\right)^{\mathrm{n}}$. The probability of missing a tumor inside a surgical sample when " $n$ " cuts were performed $\left(\mathrm{p}^{\mathrm{n}}\right)$ was calculated for all samples (Table 2). For DCIS, the average probability of missing the tumor is $87.5 \%$; therefore, DCIS should only be observed by the cancer detection techniques for $12.5 \%$ of the cases if the cross-section cuts are completely random. For invasive cancers (IDC + ILC), the average probability of missing the tumor is only $65 \%$ because the tumor sizes are larger than for DCIS; invasive cancer should only be observed by the cancer detection techniques for $35 \%$ of the cases if the cross-section cuts are completely random. Therefore, in the reported numbers for sensitivity of the manual and automated cellularity touch prep technique are always reported as minimum sensitivity, because it is hypothesized that some of the negative scores are solely due to the tumor not being sampled.

\section{Manual Analysis of Cross-Sectional Cuts}

For manual microscopy, we utilized the highest density of epithelial cells (Fig. 4a) as well as the fraction of epithelial cells as a marker of cancer. Both parameters were used to separate cancer vs. noncancer because the manual microscopy only samples less than $5 \%$ of the surface of the slide. For cases in which there are multiple cross-sectional cuts, the data from the slide with the highest cell density as determined by manual microscopy is reported. In touch prep, the tumor was pressed on the slide with minimal smearing; therefore, cells were localized in specific regions on the slide occasionally causing a manual microscopy sampling error, but this potential error is minimized by 
FIG. 3 Serial sections of cross-section of tumor. a Five serial cross-section slides of a surgical sample were performed. We found that the highest density of cells attached to slides 1,2 , and 3 , the center of the palpable tumor. b Ratio of epithelial cells (minimum divided by maximum number of epithelial cells on the serial crosssection slides) per sample. Crosssectional slides were done in cancer and normal samples; the number of crosssectional slides $(n)$ varies from two to five, showing a large variation in cell density depending in which area of the tumor was touched onto PLP-coated slides a

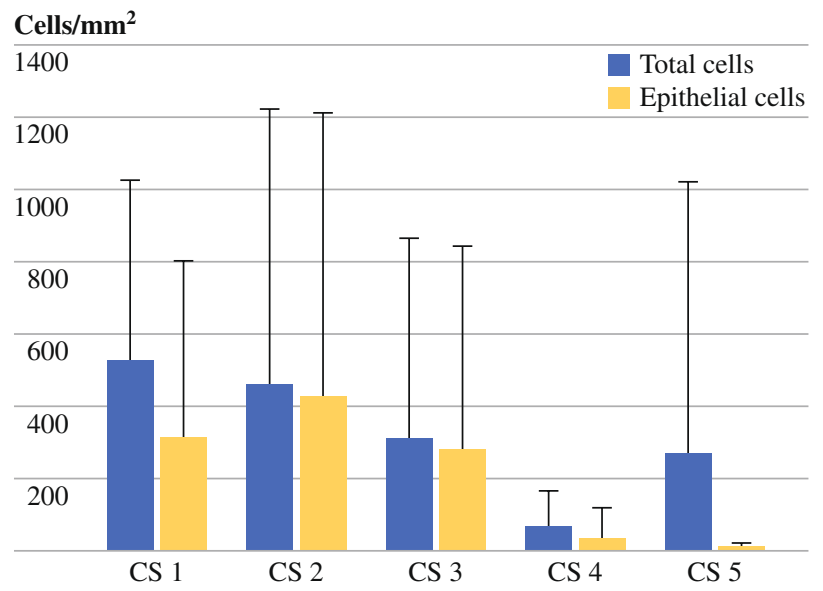

b

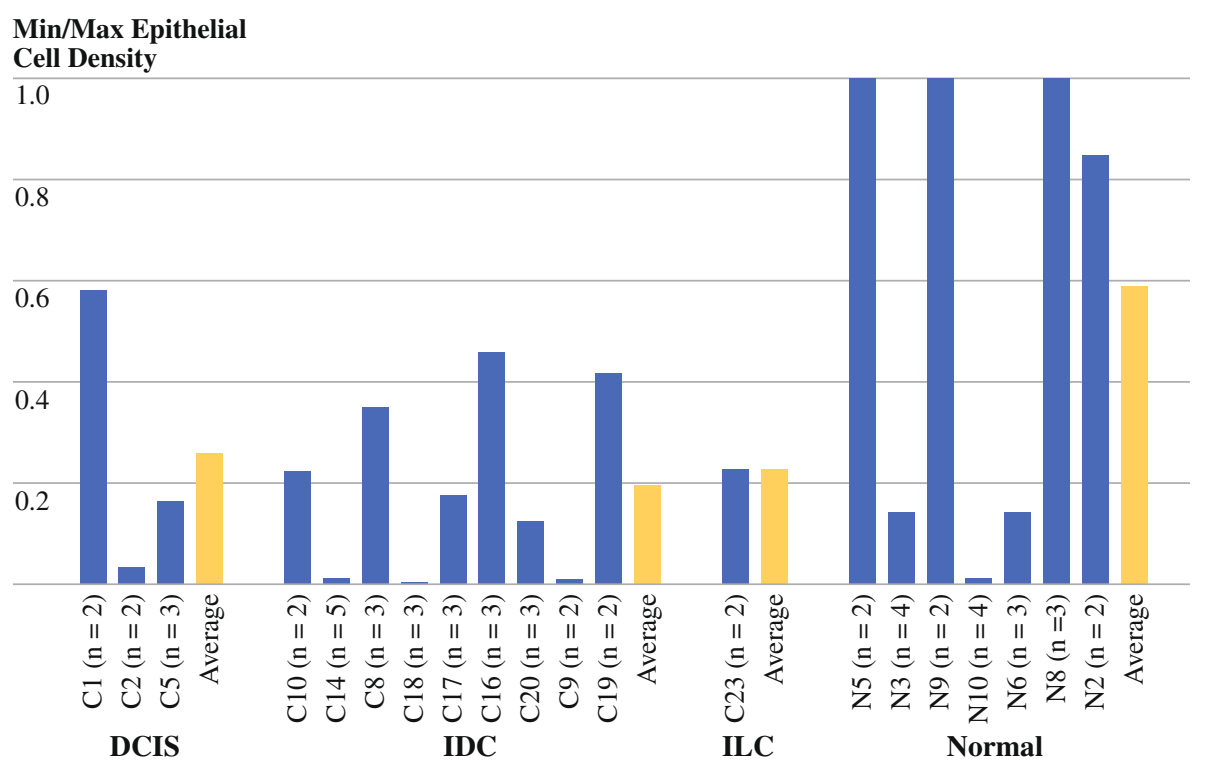

using the slide with the highest cell density. From the data in Fig. 4a, a decision boundary was drawn between cancer and noncancer samples based on Fisher linear discriminant analysis. The outlier with more than 1,000 epithelial cells/ $\mathrm{mm}^{2}$ was excluded. The Fisher analysis cutoff line is diagonal consistent with both epithelial cells density and percent epithelial cells being useful quantifiers for identifying cancer cells in manual microscopy. Samples with more than 482 epithelial cells $/ \mathrm{mm}^{2}$ or more than $80 \%$ epithelial cells were considered tumor. Based on these criteria, $64.7 \%$ (11 cases) of the invasive cases (IDC + ILC) were correctly identified as containing cancer cells, and $35.3 \%$ (6 cases) were misclassified. The four cases of IDC incorrectly classified had very high probabilities of missing the tumor because they were small tumors with few cross-sectional cuts. The calculated probabilities for the different cases were: C11 $\mathrm{p}^{\mathrm{n}}=0.97$, C12 $\mathrm{p}^{\mathrm{n}}=0.83, \mathrm{C} 15 \mathrm{p}^{\mathrm{n}}=0.56$, and C19 $\mathrm{p}^{\mathrm{n}}=0.64$. In one of these cases, no cells were found on the slide after manual inspection, indicating that this specimen was most likely misprocessed. This observation was confirmed by automatic analysis. For the three cases of ILC included in the study, only one was identified as cancer and two (C22 $\mathrm{p}^{\mathrm{n}}=0.84$, and C23 $\mathrm{p}^{\mathrm{n}}=0.58$ ) as normal. If all the invasive cases are combined, even in manual microscopy, which only samples $5 \%$ of the slide, at least $65 \%$ of the cases were correctly classified, which is double the $35 \%$ expected rate based on random cross-sectional cuts. For the six DCIS cases, $33.3 \%$ (2 cases) were correctly classified as cancer (C1 and C6), whereas four were missed (C2-C5).

\section{Automated Microscopy of Cross-Sectional Cuts}

The entire touch prep glass slide was imaged at $5 \times$ with an automated microscope, and the number of epithelial cells $/ \mathrm{mm}^{2}$ as well as the percent epithelial cells 
TABLE 2 Data of tumor and sample size, ratio between them, and probability of missing the tumor when serial cross-section slides were performed
CS cross-section

$\mathrm{p}^{n}=\left(1-\left[\mathrm{d}_{\text {tumor }} / \mathrm{d}_{\text {sample }}\right]\right) \mathrm{n}$; $n=$ number of CS slides

\begin{tabular}{llllrlll}
\hline Sample & Surgery & Diagnosis & $\begin{array}{l}\text { C-S } \\
\text { slides }\end{array}$ & \multicolumn{1}{l}{$\begin{array}{l}\text { Sample } \\
\text { size }(\mathrm{cm})\end{array}$} & $\begin{array}{l}\text { Tumor } \\
\text { size }(\mathrm{cm})\end{array}$ & $\begin{array}{l}\mathrm{d}_{\text {Tumor }} / \\
\mathrm{d}_{\text {sample }}\end{array}$ & $\mathrm{p}^{n}$ \\
\hline C2 & L & DCIS & 2 & $6 \times 4.5 \times 1.5$ & 0.6 & 0.1 & 0.81 \\
C5 & M & DCIS & 3 & $27 \times 19.5 \times 4$ & 1.5 & 0.06 & 0.84 \\
C6 & L & DCIS/LCIS & 1 & $6.4 \times 4.2 \times 1.3$ & 1 & 0.16 & 0.84 \\
C1 & L & DCIS & 2 & $5.4 \times 4.6 \times 1.6$ & 0.3 & 0.06 & 0.89 \\
C4 & M & DCIS & 1 & $23 \times 19.5 \times 9$ & 2.3 & 0.1 & 0.9 \\
C3 & M & DCIS & 1 & $23 \times 20 \times 6$ & 0.8 & 0.03 & 0.97 \\
C18 & L & IDC/ILC & 3 & $5.5 \times 4.5 \times 1.9$ & 5.5 & 1 & 0 \\
C14 & L & IDC/DCIS & 5 & $8 \times 6 \times 5$ & 2.5 & 0.31 & 0.15 \\
C8 & L & IDC & 3 & $5.3 \times 5 \times 25$ & 2.1 & 0.4 & 0.22 \\
C17 & M & IDC/DCIS & 4 & $17 \times 11 \times 3.2$ & 2.8 & 0.16 & 0.49 \\
C10 & L & IDC & 2 & $5 \times 7 \times 2.5$ & 2 & 0.29 & 0.51 \\
C15 & L & IDC/DCIS & 1 & $4.5 \times 3 \times 1.5$ & 2 & 0.44 & 0.56 \\
C19 & L & IDC/ILC & 2 & $7.5 \times 4.6 \times 2.5$ & 1.5 & 0.2 & 0.64 \\
C7 & L & IDC & 1 & $6.1 \times 4 \times 1.5$ & 1.2 & 0.2 & 0.8 \\
C9 & L & IDC & 2 & $11 \times 8 \times 3$ & 1 & 0.09 & 0.83 \\
C12 & M & IDC & 1 & $17.5 \times 17 \times 5$ & 3 & 0.17 & 0.83 \\
C16 & M & IDC/DCIS & 3 & $13.5 \times 13 \times 3$ & 0.8 & 0.06 & 0.83 \\
C13 & M & IDC & 1 & $19.5 \times 19 \times 5.5$ & 3 & 0.15 & 0.85 \\
C20 & M & IDC/DCIS & 3 & $17.5 \times 12 \times 3$ & 0.2 & 0.01 & 0.97 \\
C11 & M & IDC & 1 & $23 \times 17 \times 3$ & 0.7 & 0.03 & 0.97 \\
C21 & M & LCIS & 1 & $14.5 \times 14 \times 5.3$ & -16 & 0 & 1 \\
C23 & M & ILC & 2 & $25 \times 16 \times 35$ & 6 & 0.24 & 0.58 \\
C22 & M & ILC & 1 & $16.5 \times 13.5 \times 7$ & 2.6 & 0.16 & 0.84 \\
C24 & M & ILC & 1 & $15.5 \times 13 \times 6$ & 0.7 & 0.05 & 0.95 \\
\hline & & & & & & & \\
\end{tabular}
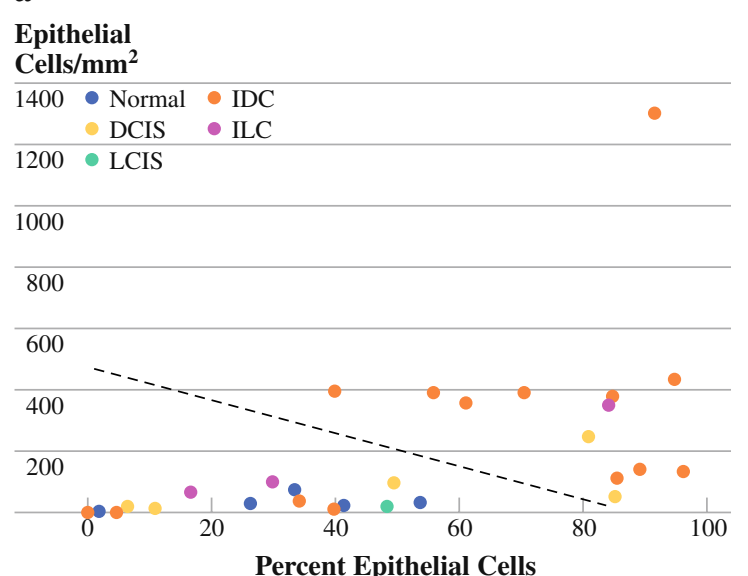

FIG. 4 Manual and automated analysis of cross-sectional cuts. a This graph shows the manual microscopy of ten fields of view across the center line. For multiple cross-sectional cuts, data from the slide with the highest epithelial cell density are displayed. High epithelial

present on the entire slide were calculated (Fig. 4b). For cases in which there are multiple cross-sectional cuts, the data from the slide with the highest epithelial cell density as determined by automated microscopy is reported; note b

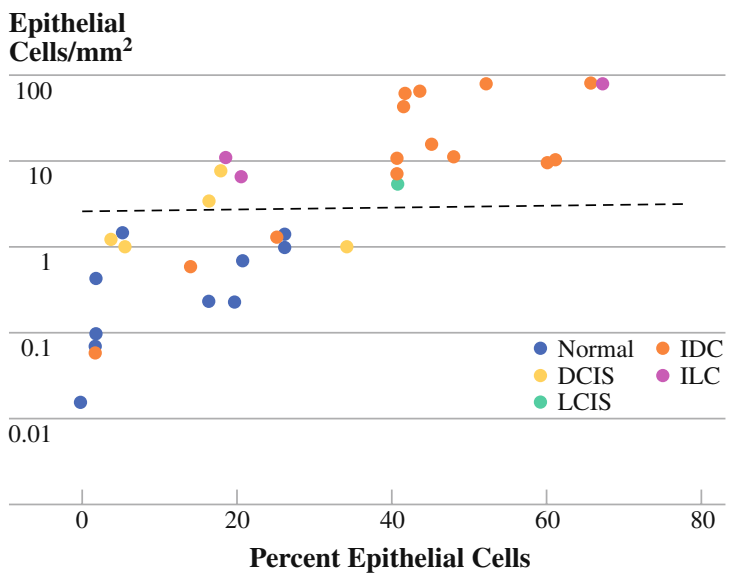

cell density or high percentage epithelial cells are consistent with cancer. b This graph demonstrates the automated analysis. Our computer program counted the total epithelial within the entire slide. We had $80 \%$ accuracy in identifying invasive cancer

this can be different than the slide with the greatest epithelial cell density as determined by manual microscopy. Furthermore, it was observed that the density of epithelial cells is much lower in automated microscopy compared 
with manual microscopy because the latter only looks a $5 \%$ of the total slide, whereas automated analysis examines the entire slide surface and therefore includes many empty areas. The density of epithelial cells is plotted on a log-scale for the automated microscopy because the dynamics range is approximately two orders of magnitude greater than for manual microscopy. From the automated microscopy data in Fig. 4b, a decision boundary was drawn between cancer and noncancer samples based on Fisher's linear discriminant analysis, which included the data points closest to the cutoff line (cancer: epithelial cells $/ \mathrm{mm}^{2}$ between 3 and 20 and noncancer: epithelial cells $/ \mathrm{mm}^{2}$ between 0.4 and 3 ). The Fisher analysis cutoff line (2.5 epithelial cells $/ \mathrm{mm}^{2}$ ) is consistent with only the epithelial cells density being required to differentiate cancer cells from noncancer cells in automated microscopy. Based on this criterion, cancer was correctly classified in at least $17(71 \%)$ cases overall: invasive plus preinvasive. For invasive cancer cancers (IDC + ILC), three cases were misclassified giving an accuracy rate of $83 \%$, which is $2.5 \times$ greater than the $35 \%$ expected rate based on random cross-sectional cuts. The automated microscopy was able to successfully detect all three ILC cases, which is important for surgeons because it is a challenge to obtain negative margins for ILC secondary to its diffuse spread throughout surrounding breast tissue. ${ }^{16}$ The three cases of IDC incorrectly classified had high probabilities of missing the tumor, because they were small tumors with few cross-sectional cuts. The calculated probabilities of missing the tumor for the different cases were: $\mathrm{C} 11 \mathrm{p}^{\mathrm{n}}=0.97, \mathrm{C} 15 \mathrm{p}^{\mathrm{n}}=0.56$, and $\mathrm{C} 19 \mathrm{p}^{\mathrm{n}}=0.64$. Note that these three IDC cases incorrectly classified by automated microscopy are a subset of the four IDC cases missed in manual microscopy, consistent with manual sampling missing the tumor. For preinvasive cancers (DCIS + LCIS), four DCIS cases were missed (C2, C3, $\mathrm{C} 4$, and C6). Two DCIS cases ( $\mathrm{C} 1$ and $\mathrm{C} 5)$ and one lobular carcinoma in situ (LCIS) were correctly classified, for an accuracy rate of $42.8 \%$ for in situ cancers, which is more than $3 \times$ the expected detection rate $(12.5 \%)$ based on random cross-sectional cuts. Examining the total number of cells counted by automated microscopy (Table 3), it is noted that epithelial cell density is the most important factor to separate cancer vs. noncancer. These results show that a nuclear stain alone cannot be used to successfully differentiate between prophylactic mastectomy tissue and cancerous tissue. The addition of an epithelial cell marker greatly enhances the ability to differentiate invasive carcinomas and lobular cancers from normal and prophylactic mastectomy tissue. Although the mean density of epithelial cells is $4 \times$ greater in DCIS than in normal samples, the standard deviation of the DCIS mean epithelial cells density
TABLE 3 Automatic microscopy cell density by tissue type

\begin{tabular}{lccc}
\hline Diagnosis & $\begin{array}{l}\text { Avg. nuclear } \\
\text { density }\end{array}$ & $\begin{array}{l}\text { Avg. epithelial } \\
\text { cell density }\end{array}$ & $\begin{array}{l}\text { Avg. total } \\
\text { density }\end{array}$ \\
\hline Normal $(n=6)$ & 1 & 1 & 1 \\
DCIS $(n=6)$ & 6 & 4.1 & 5.6 \\
IDC $(n=14)$ & 11.6 & 33.3 & 16.1 \\
Lobular $(n=3)$ & 11 & 37.1 & 16.4 \\
LCIS $(n=1)$ & 3.4 & 9.1 & 4.6 \\
Prophylactic $(n=4)$ & 10.6 & 1.1 & 8.6 \\
\hline
\end{tabular}

Number of density of epithelial vs. all other nucleated cells by tissue type is shown

$(117 \%)$ is sufficiently large that even this $4 \times$ difference is not high enough to allow epithelial cells density alone to distinguish DCIS from normal in touch prep. Additional characteristics will need to be examined to increase the recognition of DCIS. ${ }^{17}$

\section{Correlation of Automated and Manual Microscopy}

To compare the manual and automated microscopy, the epithelial cell density and the fraction of epithelial cells were compared using the slide from the cross-sectional cuts with the highest epithelial cell density as reported by automated microscopy. As shown in Fig. 5, the automated microscopy of the entire slide correlated well with manual microscopic analysis of the ten centerline fields. For epithelial cell density and percentage of epithelial cells, $\mathrm{R}^{2}$ values of 0.64 and 0.59 were observed respectively for correlation fits between automated and manual microscopy results. When few cells were present on a given slide, a poor agreement between manual counting of $5 \%$ of the slide and automated counting of the entire slide resulted in a large deviation from the trend line. In addition, even for IDC, there were occasionally cases in which a large deviation between manual and automated microscopy was observed, because manual microscopy only sampled a small fraction of the surface, which contained large cluster of cells.

The sampling variation in manual microscopy is illustrated in Fig. 6. Image tiles captured by the automated microscope were reanalyzed to mimic the manual microscopy method of examining only the centerline of a slide. All the cells were counted in each image tile and epithelial cell counts along horizontal image tile rows were summed. In slides of invasive cancers that contained large numbers of cells present in all parts of the slide, the manual and computer microscopy were equivalent. However, on slides where large numbers cells were spread randomly throughout the slide, manual analysis of a slides centerline could not accurately predict epithelial cell measurements for the entire slide. 


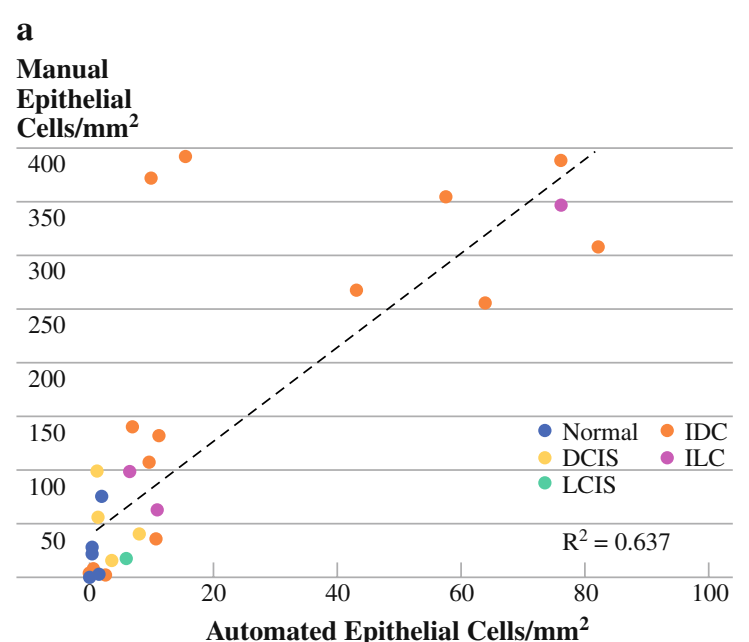

FIG. 5 Correlation of manual vs. automated analysis of the crosssections of tumor. a This graph shows a strong correlation between the manual and automated analysis based on cell density. For the manual analysis where only a fraction of the slide is analyzed, there

\section{DISCUSSION}

Obtaining negative margins during BCT operations remains a vexing problem for surgeons and patients with breast cancer. Imaging techniques, such as mammography, are detecting smaller tumors that are not palpable and are challenging to localize during surgery. Furthermore, many tumors spread along a ductal pattern and even the best imaging techniques may underestimate the amount of disease, particularly for DCIS. Intraoperatively, surgeons must make educated guesses as to the amount of tissue to remove and many times need to perform a second operation to obtain a negative microscopic margin. Furthermore, in patients with lobular cancers, it often is difficult to achieve negative margins because of their diffuse spread. ${ }^{16}$

Many techniques have been used to identify positive microscopic margins. Touch/imprint cytology has been utilized since the $1970 \mathrm{~s}$ to relatively quickly obtain pathological information on fresh specimens without disturbing the tissue for later interpretation. However, an experienced cytopathologist is necessary because correct interpretation can be challenging. ${ }^{18}$ The largest series to utilize touch preps for diagnosis and evaluation of surgical margins was completed by Klimberg et al. ${ }^{11}$ They studied 428 consecutive patients with breast masses (benign and malignant) and had 83 patients with cancer (74\% invasive and $26 \%$ DICS). Klimberg et al. performed touch prep on cross-sections to diagnose the lesion and the slides were analyzed by a cytopathologist. In their study the average tumor size was $2.2 \mathrm{~cm}$. This is relatively large and all the specimens were diagnostic excisions (no mastectomies). Therefore, the total volume of tissue removed was lower than in the present study. For the cross-section of the b

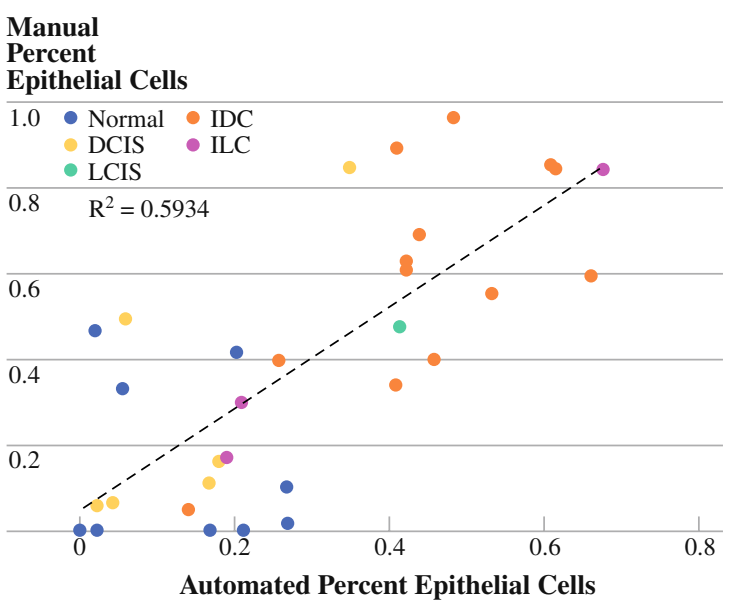

can be large variation when few cells are present on a slide. b This graph shows the correlation between manual and automated analysis based on epithelial cell density

tumor, Klimberg et al. had an accuracy rate of $99 \%$ for correctly diagnosing cancer. Klimberg et al. missed four cancers because of sampling error. At that time, the radiologist used 14- to 18-gauge cores to diagnose breast cancers, so the majority of tumor was still intact at the time of the surgical resection. ${ }^{18}$

The incidence of positive margins at University of California, San Diego, is low $(10 \%){ }^{19}$ therefore, this study utilized cross-sections of freshly excised surgical specimens. However, there were small tumors in the series, which had a high probability of not being sampled. To combat this problem, multiple cuts through surgical specimens were taken. As shown in Fig. 3b, the density of cancer cells varied by approximately $5 \times$, depending on the location of the cross-section cut; the variation may explain some false-negative cases in the cross-section study particularly in specimens of DCIS. To determine the expected accuracy of the tumor detection technique, the probability of missing the tumor in a cross-sectional cut in the absence of palpation was estimated from the size of the tumors and tissue. The average estimated probabilities of missing the tumors are $87.5 \%$ for DCIS and $35 \%$ for invasive cancer (IDC + ILC; Table 2). Therefore, the probabilities of detecting the tumors should be $12.5 \%$ for DCIS and $65 \%$ for IDC + ILC if the cross-section cuts are completely random. These two calculations explain the sampling error observed in this series. At present, radiologists use 9- to 11gauge core biopsies, which removes a large portion of the tumor; ${ }^{20}$ therefore, in the present study, the sampling error is an even larger problem for both manual and automated microscopy to obtain an accurate identification if few cells are present. Therefore, in the reported numbers for sensitivity of the automated cellularity touch prep technique are 
FIG. 6 Automated epithelial cell count by image tile row (Top-IDC, Bottom-Normal). These pictures represent the number of epithelial cells in each section of the slide. Areas of red indicate the greatest number of cells; areas of yellow indicate lower numbers of cell in any given image tile. For cases of invasive carcinoma, where many cells are present throughout the slide, manual and automated microscopy provide similar results. For slides with few cells scatter across the slide, such as with normal tissue, manual microscopy will be less accurate because there is a great deal of variation in epithelial cell density depending on which area of the slide is studied
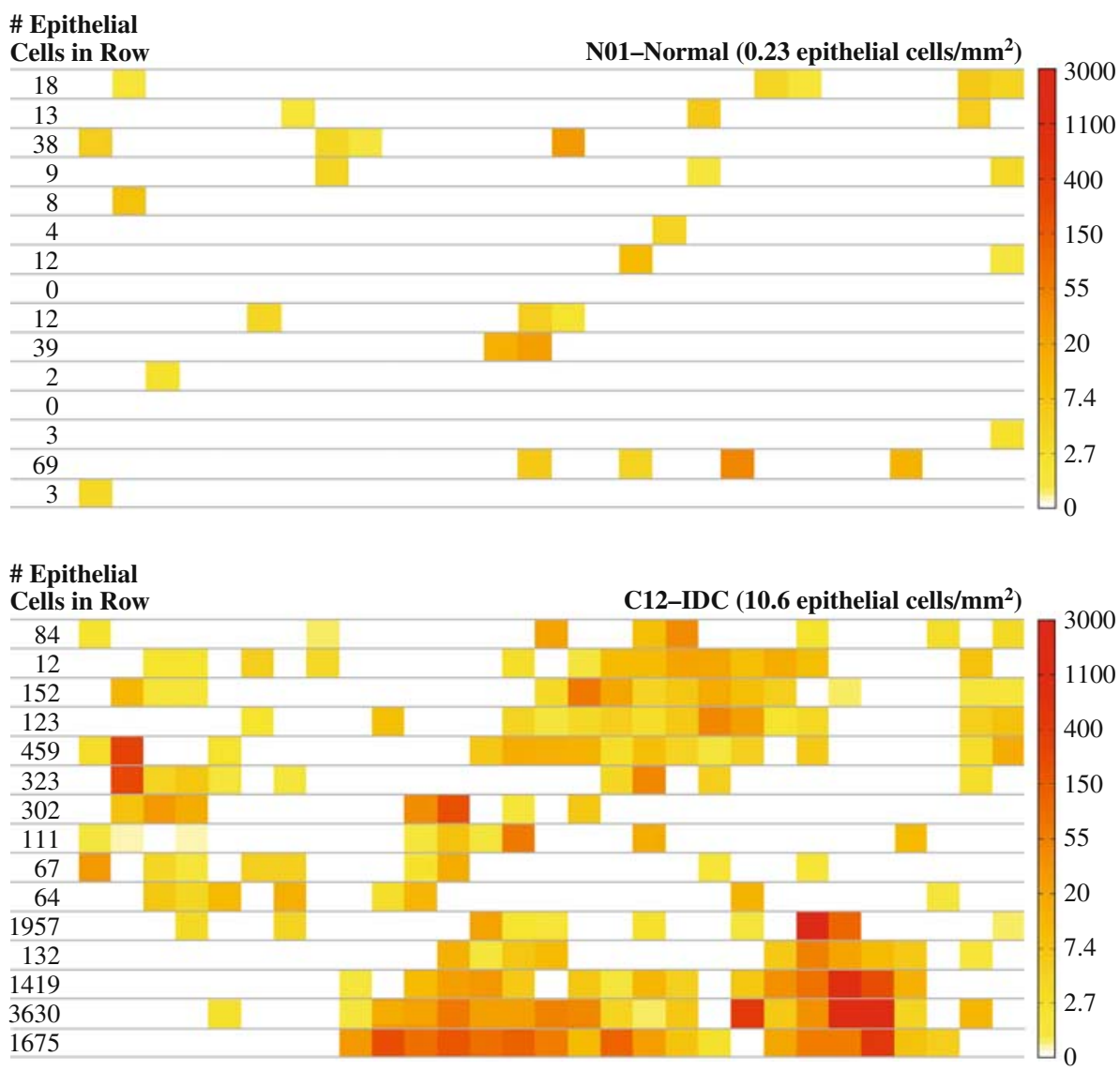

always reported as minimum sensitivity because it is hypothesized that some of the negative scores are solely due to the tumor not being sampled.

The objective of this pilot study was to create a system to evaluate surgical margins intraoperatively primarily for patients undergoing breast conservation therapy for the treatment of breast cancer. The data are consistent with the viability of ultilizing immunofluorescent staining and automated microscopy to detect cancer cells in touch preps. Confirmatory studies with larger patient populations are required to definitively prove that this automated system is reliable. The biggest challenge to surgeons is obtaining negative margins for patients undergoing breast conservation therapy but this system also may be useful for patients undergoing skin-sparing mastectomy or even nipple-preserving mastectomy where surgical margin and local recurrence is still an issue. ${ }^{21}$

Some patients with large palpable tumors or large areas of calcifications were included in this pilot project to maximize the probability of identifying the area of tumor within the breast for analysis. Many of these patients chose to undergo mastectomy because they had large palpable tumors or large areas of calcifications on mammogram. Some of the mastectomy tissue was used as part of the "normal" samples for this study, slightly increasing the difficulty of distinguishing between normal and cancer tissue because the normal tissue was from a high-risk individual with more cellular atypia. Of the 13 mastectomy cases included in this study, 4 mastectomies were prophylactic for high-risk patients (normal control tissue) and 9 mastectomies were performed due to large tumor size, multicentric disease, previous radiation, or patient preference. The 11 breast conservation cases included smaller tumors.

The preliminary results of this evaluation of the automated microscopy system show that the technique is likely to be successful and could be implemented in any hospital without special expertise in cytopathology to identify cancer cells at surgical margins for invasive breast cancer. Touch prep of the surgical margins, a rapid immunofluorescence staining protocol, and low-resolution automated microscopy can be employed to calculate the number of epithelial cells across the entire slide to differentiate invasive cancer from benign tissue with at least $83 \%$ accuracy. Eventually the technique will at least have a comparable processing and analysis time as standard frozen section. It is estimated that with a 14-megapixel camera, the setup time for automatic focus will be less than $1 \mathrm{~min}$ and the scanning time for a whole slide will be less than 5 min. 
This technique is promising to accurately identify invasive cancer cells and can be translated to evaluate the status of surgical margins. The potential of the technique to reduce reexcision can be estimated assuming that the optimized touch prep procedure would be approximately as efficient on surgical margin as it is on cross-sectional cuts. Most operations involve the use of electric cautery, which may limit the number of evaluable cells on the touch prep slides and increase the cellular fragmentation due to thermal injury. However, other investigators have successfully used hematoxylin and eosin-stained touch prep analysis on cauterized margins and newer technologies to remove breast tumors are being developed that limit thermal injury to cells. ${ }^{11,22}$ In the current small pilot study, the correct identification rates were $100 \%$ of ILC cases, $80 \%$ of IDC cases, and $40 \%$ of DCIS cases; therefore, it is estimated that the positive margin rate will cut in half when utilizing this technique. It is noted that this estimate will be lowered if the touch prep process is less efficient on heavily cauterized tissue and the estimate will be higher after correcting for sample errors in the DCIS cases.

Future studies to improve the accuracy of DCIS diagnosis will focus on using an automated microscopic analysis of the nuclear features of each cell at higher resolution; the work by Klimberg shows that this should identify even DCIS cells. This study paves the way to devise a rapid intraoperative procedure to identify breast cancer cells in excised tissue margins. It is expected that in the future, surgeons can utilize this automated microscopy system and software in the operating room and will be able to more fully evaluate the surgical margins at the time of the patient's initial operation for breast conservation surgery. This technique will reduce the necessity for multiple operations to obtain negative margins in the surgical treatment of breast cancer.

ACKNOWLEGEMENTS This research was supported by a grant form the Department of Defense Idea Grant W81XWH-06-1-052 and NIH-NCI Grant 5 U54 CA119335-03.

Open Access This article is distributed under the terms of the Creative Commons Attribution Noncommercial License which permits any noncommercial use, distribution, and reproduction in any medium, provided the original author(s) and source are credited.

\section{REFERENCES}

1. Fisher B, Anderson S, Bryant J, et al. Twenty-year follow-up of a randomized trial comparing total mastectomy, lumpectomy, and lumpectomy plus irradiation for the treatment of invasive breast cancer. N Engl J Med. 2002;347:1233-41.

2. Fisher B, Anderson S, Redmond CK, et al. Reanalysis and results after 12 years of follow-up in a randomized clinical trial comparing total mastectomy with lumpectomy with or without irradiation in the treatment of breast cancer. $N$ Engl J Med. 1995;333:1456-61.
3. van Dongen JA, Bartelink H, Fentiman IS, et al. Factors influencing local relapse and survival and results of salvage treatment after breast-conserving therapy in operable breast cancer: EORTC trial 10801, breast conservation compared with mastectomy in TNM stage I and II breast cancer. Eur J Cancer. 1992;28A:801-5.

4. Lichter AS, Lippman ME, Danforth DN Jr, et al. Mastectomy versus breast-conserving therapy in the treatment of stage I and II carcinoma of the breast: a randomized trial at the National Cancer Institute. J Clin Oncol. 1992;10:976-983.

5. Krishnan L, Stanton AL, Collins CA, et al. Form or function? Part 2. Objective cosmetic and functional correlates of quality of life in women treated with breast-conserving surgical procedures and radiotherapy. Cancer. 2001;91:2282-7.

6. Mansfield CM, Komarnicky LT, Schwartz GF, et al. Ten-year results in 1070 patients with stages I and II breast cancer treated by conservative surgery and radiation therapy. Cancer. 1995; 75:2328-36.

7. Mullenix PS, Cuadrado DG, Steele SR, et al. Secondary operations are frequently required to complete the surgical phase of therapy in the era of breast conservation and sentinel lymph node biopsy. Am J Surg. 2004;187:643-6.

8. Meric F, Mirza NQ, Vlastos G, et al. Positive surgical margins and ipsilateral breast tumor recurrence predict disease-specific survival after breast-conserving therapy. Cancer. 2003;97:926-33.

9. Singletary SE. Surgical margins in patients with early-stage breast cancer treated with breast conservation therapy. Am J Surg. 2002;184:383-93.

10. Muttalib M, Tai CC, Briant-Evans $\mathrm{T}$, et al. Intraoperative assessment of excision margins using breast imprint and scrape cytology. Breast. 2005;14:42-50.

11. Klimberg VS, Harms S, Korourian S. Assessing margin status. Surg Oncol. 1999;8:77-84.

12. Creager AJ, Shaw JA, Young PR, et al. Intraoperative evaluation of lumpectomy margins by imprint cytology with histologic correlation: a community hospital experience. Arch Pathol Lab Med. 2002;126:846-8.

13. Otsu N. A threshold selection method from grey level histograms. IEEE Trans Syst Man Cybern. 1979;9:62-6.

14. Canny J. A computational approach to edge detection. IEEE Trans Pattern Anal Mach Intell. 1986;8:679-98.

15. Cooke T, Peake M. The optimal classification using a linear discriminant for two point classes having known mean and covariance. J Multivar Anal. 2002;82:379-94.

16. Dillon MF, Hill AD, Fleming FJ, et al. Identifying patients at risk of compromised margins following breast conservation for lobular carcinoma. Am J Surg. 2006;191:201-5.

17. Gschwind R, Umbricht CB, Torhorst J, Oberholzer M. Evaluation of shape descriptors for the morphometric analysis of cell nuclei. Pathol Res Pract. 1986;181:213-22.

18. Rosenblatt R, Fineberg SA, Sparano JA, et al. Stereotactic core needle biopsy of multiple sites in the breast: efficacy and effect on patient care. Radiology. 1996;201:67-70.

19. Blair SL, Wang-Rodriguez J, Cortes-Mateos MJ, Messmer D, Sandoval S, Messmer B, et al. Enhanced touch preps improve the ease of interpretation of intraoperative breast cancer margins. Am Surg. 2007;73:973-6.

20. Povoski SP, Jimenez RE. A comprehensive evaluation of the 8gauge vacuum-assisted Mammotome ${ }^{\circledR}$ system for ultrasoundguided diagnostic biopsy and selective excision of breast lesions. World J Surg Oncol. 2007;5:83.

21. Carlson GW, Page A, Johnson E, et al. Local recurrence of ductal carcinoma in situ after skin-sparing mastectomy. J Am Coll Surg. 2007;204:1074-80.

22. Priglinger SG, Palanker D, Alge CS, et al. Pulsed electron avalanche knife: new technology for cataract surgery. $\mathrm{Br} J$ Ophthalmol. 2007;91:949-54. 